\title{
An accurate boundary value problem solver applied to scattering from cylinders with corners
}

\author{
Johan Helsing ${ }^{1}$, Anders Karlsson ${ }^{2}$ \\ ${ }^{1}$ Centre for Mathematical Sciences, \\ ${ }^{2}$ Electrical and Information Technology, \\ Lund University, Box 118, 22100 Lund, Sweden \\ ${ }^{1}$ helsing@maths.lth.se, ${ }^{2}$ anders.karlsson@eit.lth.se
}

July 30, 2018

\begin{abstract}
In this paper we consider the classic problems of scattering of waves from perfectly conducting cylinders with piecewise smooth boundaries. The scattering problems are formulated as integral equations and solved using a Nyström scheme where the corners of the cylinders are efficiently handled by a method referred to as Recursively Compressed Inverse Preconditioning (RCIP). This method has been very successful in treating static problems in non-smooth domains and the present paper shows that it works equally well for the Helmholtz equation. In the numerical examples we specialize to scattering of E- and $\mathrm{H}$-waves from a cylinder with one corner. Even at a size $k d=1000$, where $k$ is the wavenumber and $d$ the diameter, the scheme produces at least 13 digits of accuracy in the electric and magnetic fields everywhere outside the cylinder.
\end{abstract}

\section{Introduction}

The numerical simulation of scattering from cylinders has a long history in computational electromagnetics. As early as in 1881, Lord Rayleigh treated the scattering of light from a circular dielectric cylinder [1]. He considered an incident plane E-wave, i.e., the electric field is parallel to the cylinder, and 
a permittivity and permeability of the cylinder that departed only slightly from those of the surrounding medium. That enabled him to find an approximate solution that today is referred to as the Born approximation and can be viewed as spectral method solution with only one basis function, c.f. [2, Section 8.3.4]. The theory of scattering from circular cylinders and spheres, conducting or dielectric, was soon after that fully understood by using expansions of the incident and scattered waves in partial waves, c.f. [3]. Since then, a large number of papers have been published that solve scattering problems in electromagnetics, as well as in acoustics and elastodynamics, using different numerical techniques. All with the common goal of constructing faster and more accurate solvers for ever more detailed and complex geometries in two and three space dimensions. In particular, integral equation methods have become very important tools. In electromagnetics such methods were made popular by the contributions of Harrington, c.f. [4]. The mathematical foundations of the scattering problems and the integral equation formulations are discussed in the books by Colton and Kress [5, 6].

The present paper is about scattering from piecewise smooth perfectly conducting objects. The presence of boundary singularities, such as corners, tends to cause complicated asymptotics in quantities used to represent the solution. Intense mesh refinement might be needed for resolution, but this is costly and can easily lead to instabilities and the loss of precision in the computed field. In the context of integral equation solvers, regions close to the boundary are the most problematic. On the application side, scattering from non-smooth metal objects is of great importance in radar imaging of objects with sharp corners such as airplanes, vessels and vehicles. Sharp corners that are oriented perpendicular to the line of sight of a monostatic radar may create reflections that are large enough to be detected by the radar. The two-dimensional approximations can be used for elongated objects like wings but also in the evaluation of fields in the near zone of smaller objects. Other important two-dimensional problems are wave propagation in rectangular waveguides, photonic band gap structures, and substrate integrated waveguides.

The numerical solver used in this paper takes its starting point in a Fredholm second kind integral equation with integral operators that are compact away from boundary singularities and whose unknown quantity is a layer density representing the solution to the original problem. The integral equation is discretized using a Nyström scheme and composite Gauss-Legendre quadrature. At the heart of the solver lies a method called Recursively Compressed Inverse Preconditioning (RCIP). It modifies the kernels of the integral operators so that the layer density becomes piecewise smooth and simple to resolve by polynomials. Loosely speaking one can say that RCIP makes it possible 
to solve elliptic boundary value problems in piecewise smooth domains as cheaply and accurately as they can be solved in smooth domains. The RCIP method originated in 2008 [7]. In a series of papers it has been extended and successfully applied to electrostatic and elastostatic problems which, at first glance, might seem outright impossible. For example, the effective conductivity of a high-contrast conducting checkerboard with a million randomly placed squares in the unit cell was computed on a regular workstation with a relative accuracy of $10^{-9}$ [8]. A new record has been established for the three-dimensional problem of determining the capacitance of the unit cube - 13 digits compared to the seven digits that were previously known [9].

When we here apply the RCIP method to the Helmholtz equation and the exterior Dirichlet and Neumann problems we do this in a two-dimensional setting. We consider scattering of time-harmonic E- and $\mathrm{H}$-waves from an infinitely long perfectly conducting cylinder. Scattering problems are harder to solve than electrostatic problems, all other things held equal. Planar problems provide a good testing ground prior to a move up to three dimensions [10]. As we shall see, the transition from Laplace's equation to the Helmholtz equation is surprisingly straightforward and the results, presented in Section 4 below, are as good as the ones obtained for electrostatics.

Our numerical solver meets five important criteria. The first criteria is that it can handle cylinders with general shapes. In practice this means cylinders with piecewise smooth boundaries and with a finite, but arbitrary, number of corners. The second criteria is that it can treat frequencies ranging from zero up to large values of $k d$, where $k$ is the wavenumber and $d$ the diameter of the object. We have found that $k d=1000$ is quite easy to reach and for most cylinders this frequency range overlaps the frequency band where approximate high frequency methods, e.g. unified theory of diffraction in combination with physical optics, can be applied with reasonable accuracy. The third criteria is that the method can deliver accurate results for the scattered field everywhere outside the object. Even close to a corner and at $k d=1000$ the scattered field is calculated with at least 13 digits of accuracy in IEEE double precision arithmetic (16 digit precision). The fourth criteria is that the method enables fast solvers. In the present implementation the solver is fast only in the sense that the cost for modifying the kernels of the integral operators grows linearly with the number of corners in the computational domain. The method can be made fast in toto by incorporating fast multipole techniques [11, 12 or perhaps even fast direct solvers [13, 14]. The fifth criteria is that the method is automatized and flexible. It requires only a minimum of adjustments as operators and geometries change.

It is beyond the scope of the present paper to review the RCIP method in its entirety. In Section 3 we give a brief overview and a few details on 
discretization issues particular to Hankel kernels. Apart from that, we refer readers to the original research papers [7, 15, 16, 17] and to a newly written tutorial [18].

There are several recent journal papers that focus on speed and accuracy for two-dimensional scattering problems in complex geometries. In [19] scattering from two-dimensional smooth strips are treated using integral equations and a Nyström method. In [20] the approach of [19] is generalized to smooth slotted cylinders. A similar problem is treated in [21]. The schemes used in these papers give accurate results but they cannot, in a simple way, be generalized to non-smooth geometries. In [14] and in [22], on the other hand, very fast and also flexible and accurate numerical schemes are developed for the solution of integral equations modeling scattering from general objects with both corners and multi-material junctions. These papers, however, do not address the problem of accurate near field evaluation.

\section{Formulation of the problems}

We consider in-plane waves scattered by a bounded perfectly conducting cylinder with a piecewise smooth boundary $\Gamma$. The region outside the object is denoted $\Omega_{\mathrm{ex}}$, the time dependence is $e^{-\mathrm{i} \omega t}$ and $\boldsymbol{r}=(x, y)$. Both E-waves, often referred to as TM-waves, and H-waves, often referred to as TE-waves, are treated. We decompose the electric and magnetic fields into a sum of the incident field, denoted $U_{\text {inc }}(\boldsymbol{r})$, generated by a source in $\Omega_{\mathrm{ex}}$, and the scattered field, denoted $U_{\text {sca }}(\boldsymbol{r})$ in both cases.

\section{$2.1 \quad$ E-waves}

We let the electric field be parallel to the cylinder, $\boldsymbol{E}(\boldsymbol{r})=\hat{\boldsymbol{z}} U(\boldsymbol{r})$, and let $U(\boldsymbol{r})=U_{\text {inc }}(\boldsymbol{r})+U_{\text {sca }}(\boldsymbol{r})$. The scattered field $U_{\text {sca }}(\boldsymbol{r})$ satisfies the following exterior Dirichlet problem:

$$
\begin{aligned}
& \nabla^{2} U_{\text {sca }}(\boldsymbol{r})+k^{2} U_{\text {sca }}(\boldsymbol{r})=0, \boldsymbol{r} \in \Omega_{\mathrm{ex}} \\
& U_{\mathrm{sca}}(\boldsymbol{r})=-U_{\mathrm{inc}}(\boldsymbol{r}), \boldsymbol{r} \in \Gamma \\
& \lim _{|\boldsymbol{r}| \rightarrow \infty}\left(\frac{\partial}{\partial r}-\mathrm{i} k\right) U_{\mathrm{sca}}(\boldsymbol{r})=0 .
\end{aligned}
$$

We write the solution as the combined integral representation [6, eq. (3.25)].

$$
U_{\mathrm{sca}}(\boldsymbol{r})=\int_{\Gamma} \frac{\partial \Phi_{k}\left(\boldsymbol{r}, \boldsymbol{r}^{\prime}\right)}{\partial \nu_{r^{\prime}}} \rho\left(\boldsymbol{r}^{\prime}\right) \mathrm{d} \ell^{\prime}-\mathrm{i} \frac{k}{2} \int_{\Gamma} \Phi_{k}\left(\boldsymbol{r}, \boldsymbol{r}^{\prime}\right) \rho\left(\boldsymbol{r}^{\prime}\right) \mathrm{d} \ell^{\prime}, \boldsymbol{r} \in \Omega_{\mathrm{ex}},
$$


where $\Phi_{k}\left(\boldsymbol{r}, \boldsymbol{r}^{\prime}\right)=\frac{\mathrm{i}}{4} H_{0}^{(1)}\left(k\left|\boldsymbol{r}-\boldsymbol{r}^{\prime}\right|\right)$ is the free space Green function for the Helmholz equation in two dimensions, $H_{0}^{(1)}$ is the Hankel function of the first kind of order zero, and $\mathrm{d} \ell$ is an element of arc length. The index $k$ indicates that the quantity or function depends on the wavenumber $k=\omega / c$. Insertion of (4) into (2) gives the integral equation for the layer density $\rho(\boldsymbol{r})$

$$
\left(I+K_{k}-\mathrm{i} \frac{k}{2} S_{k}\right) \rho(\boldsymbol{r})=-2 U_{\mathrm{inc}}(\boldsymbol{r}), \boldsymbol{r} \in \Gamma,
$$

where

$$
\begin{aligned}
K_{k} \rho(\boldsymbol{r}) & =2 \int_{\Gamma} \frac{\partial \Phi_{k}\left(\boldsymbol{r}, \boldsymbol{r}^{\prime}\right)}{\partial \nu_{r^{\prime}}} \rho\left(\boldsymbol{r}^{\prime}\right) \mathrm{d} \ell^{\prime} \\
S_{k} \rho(\boldsymbol{r}) & =2 \int_{\Gamma} \Phi_{k}\left(\boldsymbol{r}, \boldsymbol{r}^{\prime}\right) \rho\left(\boldsymbol{r}^{\prime}\right) \mathrm{d} \ell^{\prime} .
\end{aligned}
$$

The second term on the right hand side in (4) corresponds to the term $\mathrm{i} \frac{k}{2} S_{k}$ in (5) and is added in order to ensure a unique solution for all $k$. The equation (5) is often referred to as an indirect combined field integral equation (ICFIE).

\section{$2.2 \quad$ H-waves}

We let the magnetic field be parallel to the cylinder, $\boldsymbol{H}(\boldsymbol{r})=\hat{\boldsymbol{z}} U(\boldsymbol{r})$, and let $U(\boldsymbol{r})=U_{\text {inc }}(\boldsymbol{r})+U_{\text {sca }}(\boldsymbol{r})$. The scattered field $U_{\text {sca }}(\boldsymbol{r})$ satisfies the following exterior Neumann problem

$$
\begin{aligned}
& \nabla^{2} U_{\mathrm{sca}}(\boldsymbol{r})+k^{2} U_{\mathrm{sca}}(\boldsymbol{r})=0, \boldsymbol{r} \in \Omega_{\mathrm{ex}} \\
& \frac{\partial U_{\mathrm{sca}}(\boldsymbol{r})}{\partial \nu_{\boldsymbol{r}}}=-\frac{\partial U_{\mathrm{inc}}(\boldsymbol{r})}{\partial \nu_{r}}, \boldsymbol{r} \in \Gamma \\
& \lim _{|\boldsymbol{r}| \rightarrow \infty}\left(\frac{\partial}{\partial r}-\mathrm{i} k\right) U_{\mathrm{sca}}(\boldsymbol{r})=0,
\end{aligned}
$$

where $\frac{\partial U_{\text {sca }}(\boldsymbol{r})}{\partial \nu_{r}}$ is the normal derivative of $U_{\text {sca. }}$. There are several ways to model this problem as an integral equation. We use a regularized combined field integral equation since it is always uniquely solvable. The scattered field is then obtained from the representation [23]

$$
U_{\mathrm{sca}}(\boldsymbol{r})=\int_{\Gamma} \Phi\left(\boldsymbol{r}, \boldsymbol{r}^{\prime}\right) \rho\left(\boldsymbol{r}^{\prime}\right) \mathrm{d} \ell^{\prime}+\mathrm{i} \int_{\Gamma} \frac{\partial \Phi\left(\boldsymbol{r}, \boldsymbol{r}^{\prime}\right)}{\partial \nu_{r^{\prime}}} S_{\mathrm{i} k} \rho\left(\boldsymbol{r}^{\prime}\right) \mathrm{d} \ell^{\prime}, \boldsymbol{r} \in \Omega_{\mathrm{ex}}
$$


which after insertion into (9) gives the integral equation

$$
\left(I-K_{k}^{\prime}-\mathrm{i} T_{k} S_{\mathrm{i} k}\right) \rho(\boldsymbol{r})=2 \frac{\partial U_{\mathrm{inc}}(\boldsymbol{r})}{\partial \nu_{r}} .
$$

Here $K_{k}^{\prime}$ is the adjoint to the double layer integral operator $K_{k}$ in (6)

$$
K_{k}^{\prime} \rho(\boldsymbol{r})=2 \int_{\Gamma} \frac{\partial \Phi_{k}\left(\boldsymbol{r}, \boldsymbol{r}^{\prime}\right)}{\partial \nu_{r}} \rho\left(\boldsymbol{r}^{\prime}\right) \mathrm{d} \ell^{\prime}
$$

and

$$
T_{k} \rho(\boldsymbol{r})=\frac{\partial}{\partial \nu_{\boldsymbol{r}}} K_{k} \rho(\boldsymbol{r})
$$

The equation (12) is sometimes referred to as ICFIE-R [23].

It is useful to observe that the hypersingular operator $T_{k}$ in 14 can be expressed as a sum of a simple operator and an operator that requires differentiation with respect to arc length only [24]

$$
T_{k} \rho(\boldsymbol{r})=2 k^{2} \int_{\Gamma} \Phi_{k}\left(\boldsymbol{r}, \boldsymbol{r}^{\prime}\right)\left(\boldsymbol{\nu}_{\boldsymbol{r}} \cdot \boldsymbol{\nu}_{\boldsymbol{r}^{\prime}}\right) \rho\left(\boldsymbol{r}^{\prime}\right) \mathrm{d} \ell^{\prime}+2 \frac{\mathrm{d}}{\mathrm{d} \ell} \int_{\Gamma} \Phi_{k}\left(\boldsymbol{r}, \boldsymbol{r}^{\prime}\right) \frac{\mathrm{d} \rho\left(\boldsymbol{r}^{\prime}\right)}{\mathrm{d} \ell^{\prime}} \mathrm{d} \ell^{\prime} .
$$

We may then rewrite 12 in the form

$$
\left(I+A_{k}-\mathrm{i} B_{k} S_{\mathrm{i} k}-\mathrm{i} C_{k} C_{\mathrm{i} k}\right) \rho(\boldsymbol{r})=2 \frac{\partial U_{\mathrm{inc}}(\boldsymbol{r})}{\partial \nu_{r}}, \boldsymbol{r} \in \Gamma,
$$

where $A_{k}=-K_{k}^{\prime}$ and

$$
\begin{aligned}
& B_{k} \rho(\boldsymbol{r})=2 k^{2} \int_{\Gamma} \Phi_{k}\left(\boldsymbol{r}, \boldsymbol{r}^{\prime}\right)\left(\boldsymbol{\nu}_{\boldsymbol{r}} \cdot \boldsymbol{\nu}_{\boldsymbol{r}^{\prime}}\right) \rho(\boldsymbol{r}) \mathrm{d} \ell^{\prime} \\
& C_{k} \rho(\boldsymbol{r})=2 \frac{\mathrm{d}}{\mathrm{d} \ell} \int_{\Gamma} \Phi_{k}\left(\boldsymbol{r}, \boldsymbol{r}^{\prime}\right) \rho\left(\boldsymbol{r}^{\prime}\right) \mathrm{d} \ell^{\prime}
\end{aligned}
$$

\section{Numerical scheme}

This section briefly reviews the RCIP method, for obtaining accurate solutions to integral equations on piecewise smooth surfaces, with focus on basic concepts and on some details particular to the Helmholtz equation. A richer description, along with demo codes in MATLaB, can be found in [18]. 


\subsection{Basics of the RCIP method}

Assume that we have an integral representation of a field $U(\boldsymbol{r}), \boldsymbol{r} \in \Omega_{\mathrm{ex}}$, in terms of a layer density $\rho(\boldsymbol{r})$ on a piecewise smooth boundary $\Gamma$, which leads to a Fredholm second kind integral equation

$$
(I+K) \rho(\boldsymbol{r})=g(\boldsymbol{r}), \quad \boldsymbol{r} \in \Gamma .
$$

Here $I$ is the identity, $g$ is a piecewise smooth right hand side, and $K$ is some integral operator with kernel $K\left(\boldsymbol{r}, \boldsymbol{r}^{\prime}\right)$ on $\Gamma$ that is compact away from a finite number of corners. Let us split the kernel

$$
K\left(\boldsymbol{r}, \boldsymbol{r}^{\prime}\right)=K^{\star}\left(\boldsymbol{r}, \boldsymbol{r}^{\prime}\right)+K^{\circ}\left(\boldsymbol{r}, \boldsymbol{r}^{\prime}\right)
$$

in such a way that $K^{\star}\left(\boldsymbol{r}, \boldsymbol{r}^{\prime}\right)$ is zero except for when $\boldsymbol{r}$ and $\boldsymbol{r}^{\prime}$ both lie close to the same corner vertex. In this latter case $K^{\circ}\left(\boldsymbol{r}, \boldsymbol{r}^{\prime}\right)$ is zero. The kernel split (19) corresponds to an operator split

$$
K=K^{\star}+K^{\circ}
$$

where $K^{\circ}$ is a compact operator. The variable substitution

$$
\rho(\boldsymbol{r})=\left(I+K^{\star}\right)^{-1} \tilde{\rho}(\boldsymbol{r})
$$

allows us to rewrite (18) as a right preconditioned integral equation

$$
\tilde{\rho}(\boldsymbol{r})+K^{\circ}\left(I+K^{\star}\right)^{-1} \tilde{\rho}(\boldsymbol{r})=g(\boldsymbol{r}), \quad \boldsymbol{r} \in \Gamma,
$$

where the composition $K^{\circ}\left(I+K^{\star}\right)^{-1}$ is compact.

Let us discretize (22) using a Nyström scheme with composite 16-point Gauss-Legendre quadrature. The quantities $\tilde{\rho}, K^{\circ}$, and $g$ should be simple to discretize and resolve accurately on a coarse mesh made of quadrature panels $\Gamma_{p}$ of approximately equal length. Only the inverse $\left(I+K^{\star}\right)^{-1}$ needs fine local meshes for its accurate resolution. We arrive at

$$
\left(\mathbf{I}_{\mathrm{coa}}+\mathbf{K}_{\mathrm{coa}}^{\circ} \mathbf{R}\right) \tilde{\boldsymbol{\rho}}_{\mathrm{coa}}=\mathbf{g}_{\mathrm{coa}}
$$

where the block-diagonal compressed weighted inverse matrix $\mathbf{R}$ is given by

$$
\mathbf{R}=\mathbf{P}_{W}^{T}\left(\mathbf{I}_{\text {fin }}+\mathbf{K}_{\text {fin }}^{\star}\right)^{-1} \mathbf{P} .
$$

In (23) and (24) subscript "coa" indicates a grid on the coarse mesh, subscript "fin" indicates grids on fine local meshes, the prolongation matrix $\mathbf{P}$ performs polynomial interpolation from the coarse grid to fine grids and $\mathbf{P}_{W}^{T}$ is the transpose of a weighted prolongation matrix. See [18, Section 4 and 5] for 
details. Once (23) is solved for $\tilde{\boldsymbol{\rho}}_{\text {coa }}$, a discrete weight-corrected version of the original layer density can be obtained from

$$
\hat{\boldsymbol{\rho}}_{\mathrm{coa}}=\mathbf{R} \tilde{\boldsymbol{\rho}}_{\mathrm{coa}}
$$

The solution $U(\boldsymbol{r})$ can then be recovered in most of the computational domain using $\hat{\boldsymbol{\rho}}_{\text {coa }}$ in a discretized version of the integral representation for $U(\boldsymbol{r})$.

Note that in (23), the need for resolution in corners is not visible. The transformed layer density $\tilde{\boldsymbol{\rho}}_{\text {coa }}$ on a non-smooth $\Gamma$ should be as easy to solve for as the original layer density $\boldsymbol{\rho}_{\text {coa }}$ in a discretization of (18) on a smooth $\Gamma$. All computational difficulties are concentrated in the matrix $\mathbf{R}$. Let there be $n$ discretization points on the local fine grid close to a particular corner on $\Gamma$. Judging from the definition (24), it seems as if computing $\mathbf{R}$ should be a prohibitively expensive and also unstable undertaking for large $n$. Fortunately, $\mathbf{R}$ can be computed via a fast and stable recursion which relies on a hierarchy of small nested meshes. This fast recursion enables the computation of the diagonal block of $\mathbf{R}$, that corresponds to a particular corner, at a cost only proportional to $n$. Actually, when very large $n$ are needed for resolution the cost can be further cut down with the use of Newton's method. See [18, Section 6 and 12] for details.

The fast recursion for $\mathbf{R}$ can also be run backwards for the purpose of reconstructing $\boldsymbol{\rho}_{\text {fin }}$ from $\tilde{\boldsymbol{\rho}}_{\text {coa }}$. A partial reconstruction of $\boldsymbol{\rho}_{\text {fin }}$ is needed when $U(\boldsymbol{r})$ is to be evaluated at points in $\Omega_{\mathrm{ex}}$ that lie close to corner vertices. See [18, Section 9] for details.

We remark that the integral equations (5) and (15), which are to be solved in this paper, have a more complicated appearance than the model equation (18). In practice this poses no problems for RCIP - just some extra work. The two integral operators in (5) can, for programming purposes, be combined into a single operator. The composition of integral operators in (15) can be treated with an expansion technique. With the help of two new temporary layer densities, one can arrive at a recursion for an expanded compressed inverse matrix $\mathbf{R}$ with the same structure as (24). Once $\mathbf{R}$ is computed one can extract separate blocks from it and use them in a more involved version of (23) that still uses only a single transformed global density $\tilde{\boldsymbol{\rho}}_{\text {соа }}$. See [18, Section 14 and 17] for details.

\subsection{The discretization of Hankel kernels}

High-order accurate Nyström discretization of boundary integral equations associated with the Helmholtz equation is a topic that has received much attention recently. See [25] for a comparison of various 2D schemes. We now 
present our preferred scheme by showing how to discretize the operator $K_{k}$ of (6) and the first operator on the right hand side of (4). The other integral operators of Section 2 are discretized in similar ways.

The kernel of $K_{k}$ is twice that of the first operator in (4) and can, modulo a constant of $\mathrm{i} / 2$, be expressed as

$$
K_{k}\left(\boldsymbol{r}, \boldsymbol{r}^{\prime}\right)=k\left|\boldsymbol{r}-\boldsymbol{r}^{\prime}\right| H_{1}^{(1)}\left(k\left|\boldsymbol{r}-\boldsymbol{r}^{\prime}\right|\right) \frac{\left(\boldsymbol{r}-\boldsymbol{r}^{\prime}\right) \cdot \nu_{r^{\prime}}}{\left|\boldsymbol{r}-\boldsymbol{r}^{\prime}\right|^{2}},
$$

where $H_{1}^{(1)}$ is the Hankel function of the first kind of order one. When $\boldsymbol{r} \in \Gamma$, it is instructive to write $(26)$ in the form

$$
K_{k}\left(\boldsymbol{r}, \boldsymbol{r}^{\prime}\right)=f\left(\boldsymbol{r}, \boldsymbol{r}^{\prime}\right)+\frac{2 \mathrm{i}}{\pi} \log \left|\boldsymbol{r}-\boldsymbol{r}^{\prime}\right| \Re\left\{K_{k}\left(\boldsymbol{r}, \boldsymbol{r}^{\prime}\right)\right\} .
$$

For a fixed $\boldsymbol{r} \in \Gamma$, we see from 26) and a series representation of $H_{1}^{(1)}$ that $f\left(\boldsymbol{r}, \boldsymbol{r}^{\prime}\right)$ and $\Re\left\{K_{k}\left(\boldsymbol{r}, \boldsymbol{r}^{\prime}\right)\right\}$ are smooth functions of $\boldsymbol{r}^{\prime} \in \Gamma$ and that

$$
\lim _{\boldsymbol{r}^{\prime} \rightarrow \boldsymbol{r}} \log \left|\boldsymbol{r}-\boldsymbol{r}^{\prime}\right| \Re\left\{K_{k}\left(\boldsymbol{r}, \boldsymbol{r}^{\prime}\right)\right\}=0 .
$$

Consider now the integral $I_{p}(\boldsymbol{r})$ over a quadrature panel $\Gamma_{p}$

$$
I_{p}(\boldsymbol{r})=\int_{\Gamma_{p}} K_{k}\left(\boldsymbol{r}, \boldsymbol{r}^{\prime}\right) \rho\left(\boldsymbol{r}^{\prime}\right) \mathrm{d} \ell^{\prime} .
$$

Let $\boldsymbol{r}(t)$ be a parameterization of $\Gamma$. Discretizing $K_{k}$ means being able to evaluate (29) for all $\boldsymbol{r}$ of interest, given a set of values $\rho\left(\boldsymbol{r}\left(t_{j}\right)\right)$ on each $\Gamma_{p}$.

If $\boldsymbol{r}$ is a point away from $\Gamma_{p}$, then $K_{k}\left(\boldsymbol{r}, \boldsymbol{r}^{\prime}\right)$ is a smooth function of $\boldsymbol{r}^{\prime} \in \Gamma_{p}$ and $I_{p}(\boldsymbol{r})$ can be evaluated to high accuracy using 16-point Gauss-Legendre quadrature

$$
I_{p}(\boldsymbol{r}) \approx \sum_{j} K_{k}\left(\boldsymbol{r}, \boldsymbol{r}_{j}\right) \rho_{j} s_{j} w_{j}
$$

where $\boldsymbol{r}_{j}=\boldsymbol{r}\left(t_{j}\right), \rho_{j}=\rho\left(\boldsymbol{r}\left(t_{j}\right)\right), s_{j}=\left|\mathrm{d} \boldsymbol{r}\left(t_{j}\right) / \mathrm{d} t\right|$, and $t_{j}$ and $w_{j}$ are nodes and weights on $\Gamma_{p}$.

If $\boldsymbol{r}_{i}$ is a discretization point close to $\Gamma_{p}$ or on $\Gamma_{p}$, then $K_{k}\left(\boldsymbol{r}_{i}, \boldsymbol{r}^{\prime}\right)$ is not a (sufficiently) smooth function of $\boldsymbol{r}^{\prime} \in \Gamma_{p}$ and we use (27) to arrive at

$$
I_{p}\left(\boldsymbol{r}_{i}\right) \approx \sum_{j} f\left(\boldsymbol{r}_{i}, \boldsymbol{r}_{j}\right) \rho_{j} s_{j} w_{j}+\frac{2 \mathrm{i}}{\pi} \sum_{j} \Re\left\{K_{k}\left(\boldsymbol{r}_{i}, \boldsymbol{r}_{j}\right)\right\} \rho_{j} w_{i j \mathrm{~L}}
$$

where $w_{i j \mathrm{~L}}$ are high-order product integration weights for the logarithmic operator which can be constructed using the analytic method in 16, Section 2.3]. The formula (31) can be rearranged into a particularly convenient form

$$
I_{p}\left(\boldsymbol{r}_{i}\right) \approx \sum_{j} K_{k}\left(\boldsymbol{r}_{i}, \boldsymbol{r}_{j}\right) \rho_{j} s_{j} w_{j}+\frac{2 \mathrm{i}}{\pi} \sum_{j} \Re\left\{K_{k}\left(\boldsymbol{r}_{i}, \boldsymbol{r}_{j}\right)\right\} \rho_{j} s_{j} w_{j} w_{i j \mathrm{~L}}^{\mathrm{corr}},
$$


where the weight corrections

$$
w_{i j \mathrm{~L}}^{\mathrm{corr}}=\left(\frac{w_{i j \mathrm{~L}}}{s_{j} w_{j}}-\log \left|\boldsymbol{r}_{i}-\boldsymbol{r}_{j}\right|\right)
$$

are cheap to compute and depend only on the relative length (in parameter) of neighboring quadrature panels and on nodes and weights on a canonical panel. The formula (30) with $\boldsymbol{r}=\boldsymbol{r}_{i}$ and (32) summarize our Nyström discretization of $K_{k}$ on $\Gamma$.

If $\boldsymbol{r}$ is a point not on $\Gamma$ but in $\Omega_{\mathrm{ex}}$ close to $\Gamma_{p}$, we write (26) in the form

$$
K_{k}\left(\boldsymbol{r}, \boldsymbol{r}^{\prime}\right)=g\left(\boldsymbol{r}, \boldsymbol{r}^{\prime}\right)+\frac{2 \mathrm{i}}{\pi} \log \left|\boldsymbol{r}-\boldsymbol{r}^{\prime}\right| \Re\left\{K_{k}\left(\boldsymbol{r}, \boldsymbol{r}^{\prime}\right)\right\}+\frac{2 \mathrm{i}}{\pi} \frac{\left(\boldsymbol{r}^{\prime}-\boldsymbol{r}\right) \cdot \nu_{r^{\prime}}}{\left|\boldsymbol{r}^{\prime}-\boldsymbol{r}\right|^{2}} .
$$

We see from 26$)$ and a series representation of $H_{1}^{(1)}$ that $g\left(\boldsymbol{r}, \boldsymbol{r}^{\prime}\right)$ and $\Re\left\{K_{k}\left(\boldsymbol{r}, \boldsymbol{r}^{\prime}\right)\right\}$ are smooth functions of $\boldsymbol{r}^{\prime}$. In analogy with (32) one can write

$$
\begin{aligned}
I_{p}(\boldsymbol{r}) \approx \sum_{j} K_{k}\left(\boldsymbol{r}, \boldsymbol{r}_{j}\right) & \rho\left(\boldsymbol{r}_{j}\right) s_{j} w_{j}+\frac{2 \mathrm{i}}{\pi} \sum_{j} \Re\left\{K_{k}\left(\boldsymbol{r}, \boldsymbol{r}_{j}\right)\right\} \rho\left(\boldsymbol{r}_{j}\right) s_{j} w_{j} w_{j \mathrm{~L}}^{\mathrm{corr}}(\boldsymbol{r}) \\
& +\frac{2 \mathrm{i}}{\pi} \sum_{j} \rho\left(\boldsymbol{r}_{j}\right)\left(w_{j \mathrm{C}}(\boldsymbol{r})-\frac{\left(\boldsymbol{r}_{j}-\boldsymbol{r}\right) \cdot \nu_{r_{j}}}{\left|\boldsymbol{r}_{j}-\boldsymbol{r}\right|^{2}} s_{j} w_{j}\right),
\end{aligned}
$$

where $w_{j \mathrm{~L}}^{\mathrm{corr}}(\boldsymbol{r})$ are weight corrections as in (33), but with $\boldsymbol{r}_{i}$ replaced by $\boldsymbol{r}$, and $w_{j \mathrm{C}}(\boldsymbol{r})$ are high-order product integration weights for the Cauchy singular operator which can be constructed using the analytic method in [16, Section 2.1]. The formulas (30) and (35) are used to discretize the first operator in (4) when producing field plots.

\subsection{Convergence and error estimates}

Our solver shows a stable behavior. This means that the solution converges rapidly with coarse mesh refinement up until a point beyond which no further improvement occurs. Actually, beyond this optimal point there will be a slow decay in the quality of the solution, due to accumulated roundoff error. The precise location of the optimal point is hard to determine a priori. It depends on the geometry, on the boundary conditions, and on the wave number. The optimal point is determined experimentally in the numerical examples of Section 4 .

We have estimated the accuracy in our solutions $U(\boldsymbol{r})$ rather thoroughly. The tutorial [18, Section 18] contains error plots for exterior problems in non-smooth domains produced in a direct way. These are achieved by generating the boundary conditions on $\Gamma$ via line sources inside $\Gamma$ so that the exact 
solution is known. In the plane-wave scattering examples of Section 4.1, below, no exact results are known. Therefore we proceed as follows: we first compute a solution $U(\boldsymbol{r})$ using a number of coarse panels on $\Gamma$ deemed sufficient for resolution. Then we increase this number with $50 \%$ and solve again. The difference between the resolved value of $U(\boldsymbol{r})$ and the overresolved value of $U(\boldsymbol{r})$ is used as an indirect pointwise error estimate. Yet an indirect method to estimate the (overall) precision in the computations is by comparing the scattering cross section computed from its definition (close to $\Gamma$ ) with its value obtained via the optical theorem (at infinity). See, further, Section 4.2, As it turns out, the various error estimates seem to agree well.

\section{$4 \quad$ Numerical examples}

We shall now solve (5) and (15) for the unknown density $\rho(\boldsymbol{r})$, using the method of Section 3 , and then evaluate the scattered fields of (4) and (11). We restrict the numerical examples to scattering from an infinite straight cylinder with boundary $\Gamma$ described by

$$
\boldsymbol{r}(t)=\sin (\pi t)(\cos ((t-0.5) \pi / 2), \sin ((t-0.5) \pi / 2)), \quad t \in[0,1],
$$

and to the incident plane wave $U_{\text {inc }}(\boldsymbol{r})=e^{\mathrm{i} k y}$ for both E-waves and H-waves. The object parameterized in (36) has a corner with opening angle $\theta=\pi / 2$ at $\boldsymbol{r}=0$ and a diameter $d=1$, in arbitrary length units, so that $k d=k$. The examples cover sizes from $k d=1$ up to $k d=1000$. We have seen that at $k d=1000$ the frequency is high enough such that the uniform theory of diffraction theory can be applied. All numerical examples are executed in MATLAB on a workstation equipped with an IntelXeon E5430 CPU at 2.66 $\mathrm{GHz}$ and $32 \mathrm{~GB}$ of memory.

\subsection{Near field}

A criterion for a powerful method is that it should be able to calculate the electric and magnetic fields everywhere in $\Omega_{\mathrm{ex}}$. Figures 1 and 2 show the total electric field for the E-wave and total magnetic field for the $\mathrm{H}$-wave in the vicinity of the scattering object and the corresponding errors. The scattering object itself appears in green color in the left images and in white color in the right images. The number of spatial points in each image is $10^{6}$. It is encouraging to see, in the right images of Figures 1 and 2, that the accuracy is high even close to the boundary and, in particular, close to the corner. 

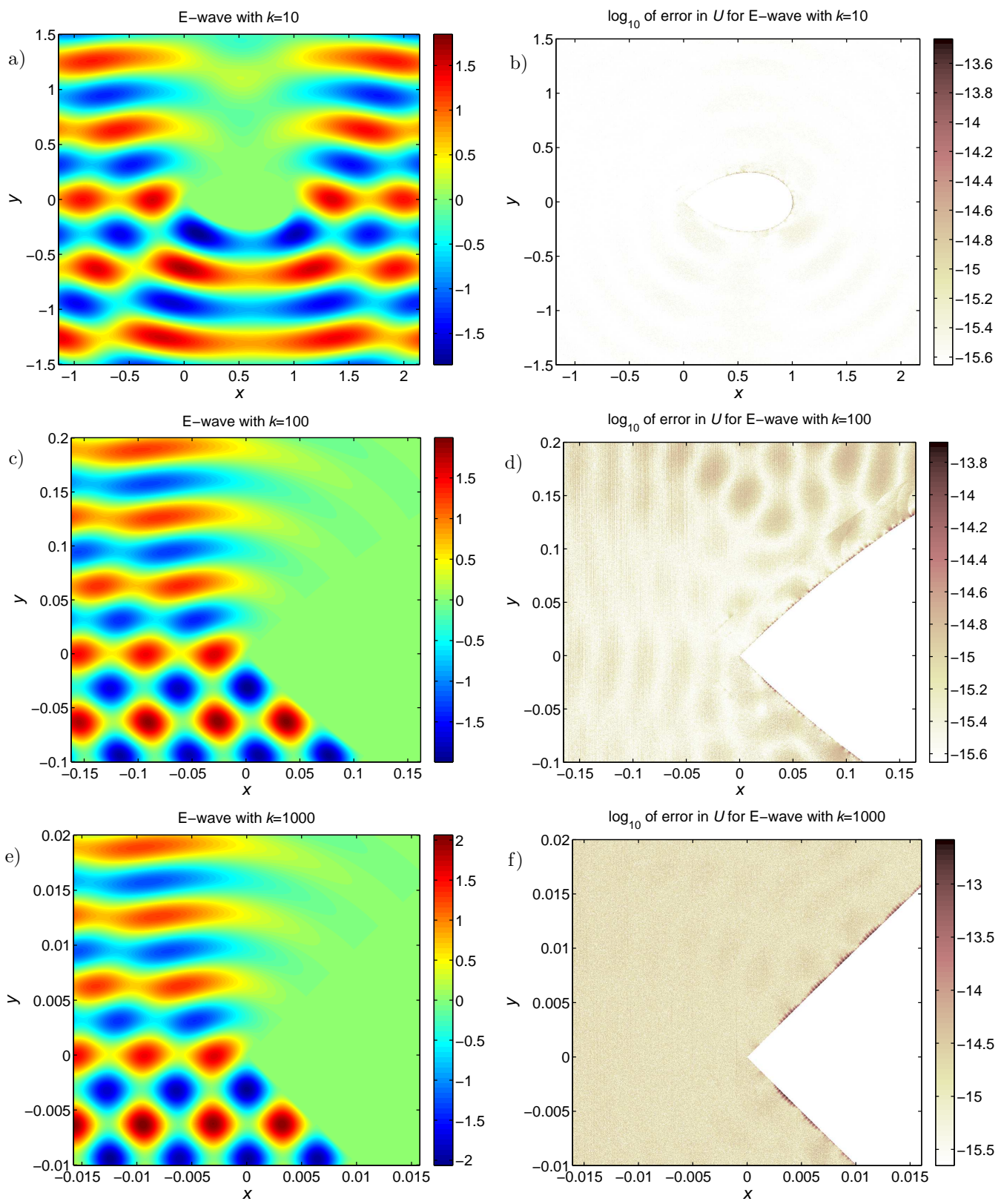

Figure 1: Left: a), c), e) show $\Re\{U(\boldsymbol{r})\}$ for a plane E-wave $U_{\text {inc }}(\boldsymbol{r})=e^{\mathrm{i} k y}$ incident on the perfectly conducting cylinder with boundary $\Gamma$ given by (36). Right: b), d), f) show absolute errors. 

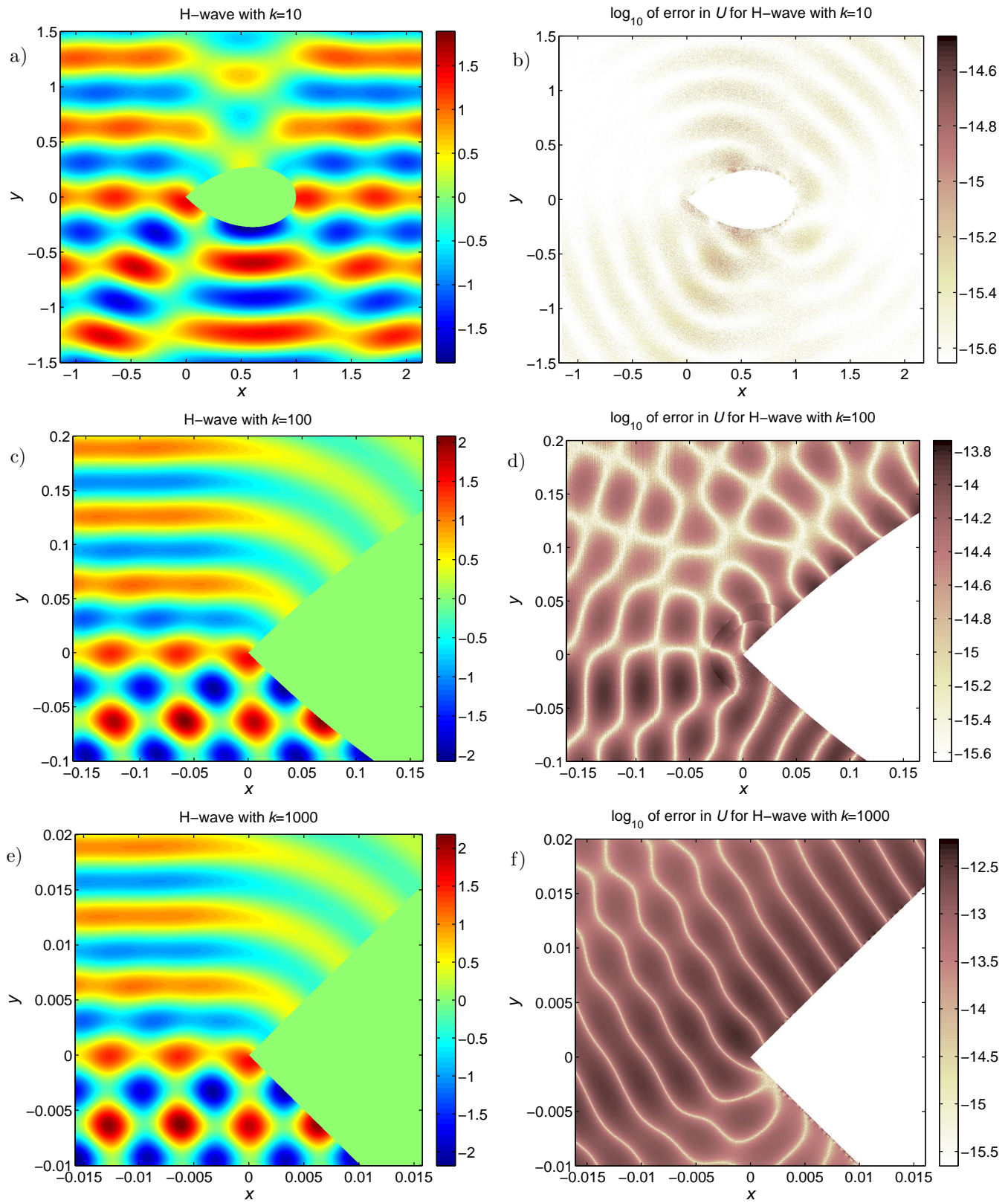

Figure 2: Left: a), c), e) show $\Re\{U(\boldsymbol{r})\}$ for a plane H-wave $U_{\text {inc }}(\boldsymbol{r})=e^{\mathrm{i} k y}$ incident on the perfectly conducting cylinder with boundary $\Gamma$ given by (36). Right: b), d), f) show absolute errors. 
The integrals in (4) and (11) are often thought of as difficult to evaluate close to the boundary due to the singularities in the Hankel functions when $\boldsymbol{r}^{\prime}=\boldsymbol{r}$. However, the present method circumvents these problems using the high-order analytic quadrature outlined in Section 3.2 .

In Figures 1 a), c), e) the real part of the total electric field $U(\boldsymbol{r})$ for the E-wave case is plotted for $k d=10,100$, and 1000. To capture the diffraction pattern in the vicinity of the corner, the field is plotted in a rectangular region with side length proportional to $1 / k$ and center at the tip of the corner. At $k d=10$ the error is very small, as seen from Figure $1 \mathrm{~b}$ ). The errors increase slightly with $k d$ but even at $k d=1000$ we get 14 digits or better almost everywhere, as depicted in Figure 1 f). For H-waves the accuracy is almost as good as for the E-waves, as seen from Figure 2.

For $k d=100$ and 1000 we can interpret the field plots in Figures 1 c), e) and 2 c), e) through the theory of diffraction. Thus, the outer region $\Omega_{\mathrm{ex}}$ is divided into three subregions separated by the reflection boundary and the shadow boundary.

\subsection{Scattering cross section and optical theorem}

In two dimensions the scattering cross section reads

$$
\sigma_{\mathrm{sca}}=\frac{P_{\mathrm{sca}}}{\boldsymbol{S}_{\mathrm{inc}} \cdot \hat{\boldsymbol{y}}}=\Re\left\{\frac{\mathrm{i}}{\omega} \int_{\Gamma_{\mathrm{circ}}} U_{\mathrm{sca}}\left(\boldsymbol{r}^{\prime}\right) \frac{\partial U_{\mathrm{sca}}^{*}\left(\boldsymbol{r}^{\prime}\right)}{\partial \nu_{r^{\prime}}} \mathrm{d} \ell^{\prime}\right\},
$$

where $P_{\text {sca }}$ is the scattered power per unit length, $\boldsymbol{S}_{\text {inc }} \cdot \hat{\boldsymbol{y}}$ is the $y$-component of the Poynting vector of the incident field, i.e. the incident power density, the boundary $\Gamma_{\text {circ }}$ is a closed curve that circumscribes the boundary $\Gamma$, and the star denotes complex conjugation. The expression holds for both E- and $\mathrm{H}$-waves. In a numerical experiment with the cylinder of (36) we let $\Gamma_{\text {circ }}$ be a circle of radius 0.55 and with center at $\boldsymbol{r}=(0.5,0)$. Since the diameter of the scatterer is $d=1$, the smallest distance between the $\Gamma$ and $\Gamma_{\text {circ }}$ is 0.05 and it occurs at the corner vertex and at a point opposite to the corner vertex. For evaluation points $\boldsymbol{r}^{\prime}$ so close to the boundary, the field $U_{\text {sca }}\left(\boldsymbol{r}^{\prime}\right)$ and its normal derivative are in general hard to evaluate. But, as we have already seen in Section 4.1, the RCIP method and the high-order analytic quadrature outlined in Section 3.2 should allow for high accuracy.

By utilizing the optical theorem we get an alternative expression for the scattering cross section

$$
\sigma_{\text {sca }}=-\lim _{y \rightarrow \infty} \Re\left\{\frac{4}{\omega} U_{\text {sca }}(0, y) \sqrt{\frac{\pi \omega y}{2}} e^{-\mathrm{i}(\omega y-\pi / 4)}\right\}
$$



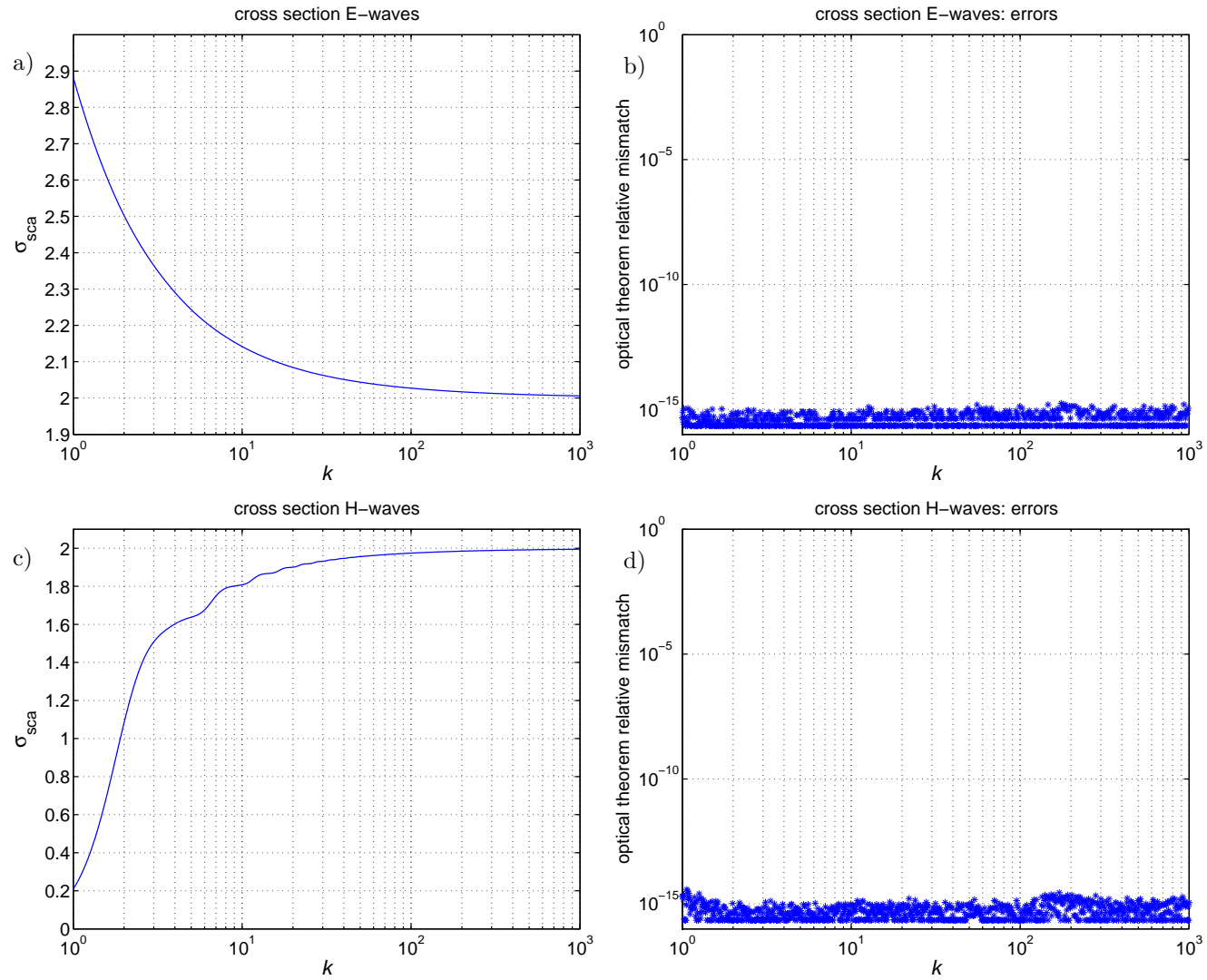

Figure 3: The scattering cross sections $\sigma_{\text {sca }}$ for the E-wave, a), and $\mathrm{H}$-wave, c), calculated by the optical theorem (38) and the relative error, b) and d), compared to the values from equation (37) 
which should be even simpler to evaluate than (37) since it only involves the far field. The mismatch between the scattering cross sections computed via (37) and via (38) can be used as an error estimate for both expressions. The cross sections for the E-waves along with such error estimates are given in Figure 3 a) and b) and the corresponding data for the $\mathrm{H}$-waves are given in Figures 3 c) and d). The mismatch error is on the order of $10^{-15}$. The cross sections in Figures 3 a) and c) show the well known behaviors for large and small values of $k$.

\section{Conclusions}

We have shown how the basic problems of scattering of E- and H-waves from perfectly conducting cylinders with corners can be solved numerically to high accuracy on a mesh that on a global level is not refined close to corner vertices. We give examples where the scattered electric and magnetic fields from a cylinder with one corner and with a diameter of up to 160 wavelengths is obtained with 14 digits of accuracy almost everywhere outside the cylinder. This success is achieved by

1. choosing a suitable integral representation of the scattered field in terms of an unknown layer density

2. formulating the scattering problem as a Fredholm second kind integral equation with operators that are compact away from the corners

3. discretizing using a Nyström scheme and a mix of composite GaussLegendre quadrature and high-order analytic product rules

4. modifying the discretized integral equation so that the new unknown, a transformed layer density, is piecewise smooth

5. solving the resulting well-conditioned linear system iteratively for the transformed layer density

6. partially reconstructing the original layer density from the transformed layer density

7. evaluating the scattered field from a discretization of its integral representation which, again, relies on a mix of composite Gauss-Legendre quadrature and high-order analytic product rules

While some steps in this scheme are standard, step 4, 6, and 7 are unique to the recently developed RCIP method. Conceptually, step 4 and 5 correspond 
to applying a fast direct solver [26] locally to regions with troublesome geometry and then applying a global iterative method. This gives us many of the advantages of fast direct methods, for example the ability to deal with certain classes of operators whose spectra make them unsuitable for iterative methods. In addition, this approach is typically much faster than using only a fast direct solver.

Our numerical scheme can be extended to related problems of importance in e.g. band-gap structures, axially symmetric cavities for accelerators, and remote sensing of underground objects. Thus we can extend the method to scattering from homogeneous dielectric cylinders, scattering from multiple cylinders, scattering from cylinders in layered structures (c.f. [27]), scattering of plane waves at oblique angles from cylinders, and scattering from axially symmetric three-dimensional geometries. Some of these problems will be addressed in forthcoming papers.

\section{Acknowledgment}

This work was supported in part by the Swedish Research Council under contract 621-2011-5516.

\section{References}

[1] L. Rayleigh, "On the electromagnetic theory of light," Phil. Mag. 12, 81 (1881).

[2] J. van Bladel, Electromagnetic Fields (Hemisphere Publication Corporation, New York, 1986). Revised Printing.

[3] G. Mie, "Beiträge zur Optik trüber Medien, speziell kolloidaler Metallösungen," Ann. Phys. Leipzig 25, 377-445 (1908).

[4] R. F. Harrington, Field Computation by Moment Methods (Macmillan, New York, 1968).

[5] D. Colton and R. Kress, Integral Equation Methods in Scattering Theory (John Wiley \& Sons, New York, 1983).

[6] D. Colton and R. Kress, Inverse Acoustic and Electromagnetic Scattering Theory (Springer-Verlag, Berlin, 1992).

[7] J. Helsing and R. Ojala, "Corner singularities for elliptic problems: Integral equations, graded meshes, quadrature, and compressed inverse 
preconditioning," Journal of Computational Physics 227, 8820 - 8840 (2008).

[8] J. Helsing, "The effective conductivity of arrays of squares: Large random unit cells and extreme contrast ratios," Journal of Computational Physics 230, 7533 - 7547 (2011).

[9] J. Helsing and K.-M. Perfekt, "On the polarizability and capacitance of the cube," Applied and Computational Harmonic Analysis (in press 2012).

[10] A. Klöckner, A. Barnett, L. Greengard, and M. O’Neil, "Quadrature by Expansion: A New Method for the Evaluation of Layer Potentials," arXiv:1207.4461 (2012).

[11] J. Carrier, L. Greengard, and V. Rokhlin, "A fast adaptive multipole algorithm for particle simulations," SIAM Journal on Scientific and Statistical Computing 9, 669-686 (1988).

[12] H. Cheng, W. Crutchfield, Z. Gimbutas, L. Greengard, J. Huang, V. Rokhlin, N. Yarvin, and J. Zhao, "Remarks on the implementation of the wideband FMM for the Helmholtz equation in two dimensions," Contemporary Mathematics 408, 99 (2006).

[13] P. Martinsson and V. Rokhlin, "A fast direct solver for boundary integral equations in two dimensions," Journal of Computational Physics 205, $1-23(2005)$.

[14] J. Bremer, "A fast direct solver for the integral equations of scattering theory on planar curves with corners," Journal of Computational Physics 231, 1879-1899 (2012).

[15] J. Helsing and R. Ojala, "Elastostatic computations on aggregates of grains with sharp interfaces, corners, and triple-junctions," International Journal of Solids and Structures 46, 4437 - 4450 (2009).

[16] J. Helsing, "Integral equation methods for elliptic problems with boundary conditions of mixed type," Journal of Computational Physics 228, $8892-8907$ (2009).

[17] J. Helsing, "A fast and stable solver for singular integral equations on piecewise smooth curves," SIAM Journal on Scientific Computing 33, 153-174 (2011). 
[18] J. Helsing, "Solving integral equations on piecewise smooth boundaries using the RCIP method: a tutorial," arXiv:1207.6737 (2012).

[19] J. L. Tsalamengas, "Exponentially converging Nyström methods in scattering from infinite curved smooth strips-Part 1:TM-case," IEEE Trans. Antennas Propagat. 58, 3265-3274 (2010).

[20] J. L. Tsalamengas and C. V. Nanakos, "Nyström solution to oblique scattering of arbitrarily polarized waves by dielectric-filled slotted cylinders," IEEE Trans. Antennas Propagat. 60, 2802-2813 (2012).

[21] M. S. Tsong and W. C. Chew, "Nyström method with edge condition for electromagnetic scattering by 2D open structures," Progress in Electromagnetics Research 62, 49-68 (2006).

[22] L. Greengard and J.-Y. Lee, "Stable and accurate integral equation methods for scattering problems with multiple material interfaces in two dimensions," Journal of Computational Physics 231, 2389 - 2395 (2012).

[23] O. Bruno, T. Elling, and C. Turc, "Regularized integral equations and fast high-order solvers for sound-hard acoustic scattering problems," International Journal for Numerical Methods in Engineering 91, 10451072 (2012).

[24] R. Kress, "On the numerical solution of a hypersingular integral equation in scattering theory," Journal of Computational and Applied Mathematics 61, $345-360$ (1995).

[25] S. Hao, P. G. Martinsson, and P. Young, "High-order accurate Nystrom discretization of integral equations with weakly singular kernels on smooth curves in the plane," arXiv:1112.6262 (2011).

[26] W. Y. Kong, J. Bremer, and V. Rokhlin, "An adaptive fast direct solver for boundary integral equations in two dimensions," Applied and Computational Harmonic Analysis 31, 346 - 369 (2011).

[27] A. Karlsson, "Scattering from inhomogeneities in layered structures," The Journal of the Acoustical Society of America 71, 1083-1092 (1982). 